\title{
Theoretical Comparison between the Flicker Noise Behavior of Graphene and of Ordinary Semiconductors
}

\author{
Massimo Macucci iD and Paolo Marconcini \\ Dipartimento di Ingegneria dell'Informazione, Universita di Pisa, Via Girolamo Caruso 16, 56122 Pisa, Italy \\ Correspondence should be addressed to Paolo Marconcini; p.marconcini@iet.unipi.it
}

Received 4 January 2020; Accepted 31 January 2020; Published 9 March 2020

Guest Editor: Laszlo B. Kish

Copyright ( 2020 Massimo Macucci and Paolo Marconcini. This is an open access article distributed under the Creative Commons Attribution License, which permits unrestricted use, distribution, and reproduction in any medium, provided the original work is properly cited.

\begin{abstract}
Graphene is a material of particular interest for the implementation of sensors, and the ultimate performance of devices based on such a material is often determined by its flicker noise properties. Indeed, graphene exhibits, with respect to the vast majority of ordinary semiconductors, a peculiar behavior of the flicker noise power spectral density as a function of the charge carrier density. While in most materials flicker noise obeys the empirical Hooge law, with a power spectral density inversely proportional to the number of free charge carriers, in bilayer, and sometimes monolayer, graphene a counterintuitive behavior, with a minimum of flicker noise at the charge neutrality point, has been observed. We present an explanation for this stark difference, exploiting a model in which we enforce both the mass action law and the neutrality condition on the charge fluctuations deriving from trapping/detrapping phenomena. Here, in particular, we focus on the comparison between graphene and other semiconducting materials, concluding that a minimum of flicker noise at the charge neutrality point can appear only in the presence of a symmetric electron-hole behavior, a condition characteristic of graphene, but which is not found in the other commonly used semiconductors.
\end{abstract}

\section{Introduction}

In the actual operation of electronic devices, random fluctuations (the "noise") are always superimposed to the deterministic electrical quantity (the "signal") which conveys the desired information. Such fluctuations derive from underlying microscopic phenomena and in sensors they may limit the achievable sensitivity or, as in the case of fluctuationenhanced sensing [1], contribute to the improvement of sensor performance, in particular in terms of selectivity. Several forms of random electrical fluctuations may exist in electronic devices: shot noise, thermal noise, generationrecombination noise, burst noise, and $1 / f$ (flicker) noise, just to cite the main ones [2-9]. They differ for their physical origin and for the dependence of their power spectral density on the physical parameters characterizing the device operation, such as temperature, frequency, bias current, charge density, and material resistivity.
In particular, understanding the physical mechanisms that govern the intrinsic noise of electronic devices, and in particular of sensors, can significantly help the designer in the choice of the best material for their fabrication and of the optimal bias point for their operation.

Here, we focus on $1 / f$ (flicker) noise, so called from the behavior of its power spectral density, which is inversely proportional to the frequency. This type of noise mainly originates from trapping and detrapping of charge carriers, due to impurities (traps) located inside or near the channel where the device current flows. In general, its amplitude is therefore proportional to the trap concentration.

The power spectral density of the $1 / f$ current noise is often approximated with the empirical Hooge formula [10]:

$$
S_{I}=\frac{\alpha_{\mathrm{H}} I^{2}}{N f}
$$


where $\alpha_{H}$ is Hooge's constant, $I$ is the mean bias current, $N$ is the number of carriers in the device, and $f$ is the frequency. According to this formula, $1 / f$ noise should be greater when the number $N$ of carriers in the device is lower. This is actually what is generally observed in ordinary semiconductors $[11,12]$, such as silicon or gallium arsenide. However, a different behavior has been observed in measurements performed on graphene samples.

Monolayer graphene is a recently isolated material [13] made up by a planar hexagonal lattice of carbon atoms. It is a semiconductor with a zero energy gap [14-19], even though an energy gap can be introduced, for example, by lateral confinement [20-22], strain [23, 24], doping [25-27], functionalization [28, 29], or introducing a lattice of antidots in the material layer [30, 31]. Its dispersion relations around the degeneration points between conduction and valence bands (the so-called Dirac points, i.e., the charge neutrality points) are linear and thus in graphene charge carriers present a zero effective mass. Since its isolation from graphite, it has been the focus of a large research effort (which has more recently extended to a wider family of two-dimensional materials [32-34]), because it possesses very attractive electrical, thermal, optical, and mechanical properties [35-47]. Moreover, graphene exhibits very uncommon physical phenomena [48-50], typical of relativistic mechanics, because its effective mass transport equation coincides with the Dirac equation $[18,51,52]$ (the wave equation which describes relativistic spin-1/2 particles). Bilayer graphene is instead made up of two coupled graphene layers [14, 15, 53-56]. Bilayer graphene with Bernal stacking has nonlinear dispersion relations with a zero gap; however, an energy gap can be easily induced applying an orthogonal electric field, which introduces a shift between the electrochemical potentials of the two layers.

Graphene is of particular interest for the implementation of sensors because of its quasi-two-dimensional nature, which leads to a very large surface-to-volume ratio: since the interaction of a sensor with an analyte mainly occurs as a result of surface adsorption, while conduction is a bulk property, a large surface-to-volume ratio will lead to a large relative variation of resistance when the sensor interacts with the analyte.

In graphene, measurements of flicker noise power spectral density as a function of the charge density (usually tuned adjusting a gate voltage) have shown a variety of different behaviors [57-70]. While in most monolayer samples a " $\Lambda$ "-shaped behavior was observed [60-63], analogous to that of common semiconductors, in suspended monolayer graphene and in most bilayer samples an "M"-shaped (or "V"-shaped) behavior was measured, with a local minimum of flicker noise near the Dirac point, where the carrier density is lowest [58, 59, 61-69], in contrast with what would be expected from Hooge's formula. Several explanations have been proposed to understand this behavior [61, 62, 64-66, 68].

An interesting theory [71] exploited the electrostatic screening of the trapped carriers and the peculiar properties of the graphene band structure to explain the observed fea- tures. We have developed a different approach [72] that leads to analogous results, exploiting a model based on the conservation of charge neutrality and on the mass action law (which has to be satisfied if the main fluctuations in flicker noise are slow compared to the generation-recombination times of carriers).

Here, we extend this model to the case of generic semiconductors, and we use it to explain the origin of this discrepancy between the behavior observed in graphene samples and that typical of common semiconductors. In particular, we show that a minimum of the $1 / f$ noise at the charge neutrality point is expected in materials (such as graphene) where electrons and holes have an identical mobility, in the presence of a quite low potential disorder. This is actually the case of suspended graphene or of bilayer graphene (where the electrostatic effect of randomly located charged impurities is strongly screened). In common semiconductors, where the electron and hole behavior is in general different $[50,73]$, this local minimum in the flicker noise power spectral density is much less apparent and is totally suppressed by the inevitable presence of potential disorder.

\section{Simulation Model}

In order to relate the (microscopic) phenomena, consisting in the motion of the charge carriers and their capture and reemission by the traps to the (macroscopic) currents at the terminals of the device, we can use the Ramo-Shockley theorem $[74,75]$ (then generalized by Pellegrini with the electrokinematics theorem $[71,76,77])$. We assume the scattering phenomena to take place on timescales much faster than the considered trapping/detrapping events, in such a way as to be able to actually define a drift current. The current at the terminals is given by

$$
\begin{aligned}
i & =\int_{A} q\left(-\vec{v}_{n} n(x, y)+\vec{v}_{p} p(x, y)\right) \cdot \vec{F}(x, y) d x d y \\
& =\int_{A} q\left(\mu_{n} n(x, y)+\mu_{p} p(x, y)\right) \vec{E}(x, y) \cdot \vec{F}(x, y) d x d y,
\end{aligned}
$$

where $q$ is the modulus of the elementary charge; $\vec{v}_{n}$ and $\vec{v}_{p}$ are the drift velocities of electrons and holes, respectively; $\vec{F}$ $(x, y)$ is the electric field that would be produced in the point with coordinates $(x, y)$ by a unit potential applied to the electrode for which we want to compute the current (while the other electrode is grounded and in the absence of mobile charges) [74]; $\vec{E}(x, y)$ is the electric field actually present in the device; $\mu_{n}\left(\mu_{p}\right)$ and $n(p)$ are the mobilities and surface densities of electrons (holes); and $A$ is the area of the device, equal to the product of the width $W$ and the length $L$. Since $\vec{F}(x, y)$ is an electric field per unit applied potential, thus the ratio of an electric field to the applied potential, it has the dimension of the reciprocal of a length. The actual functional dependence of $\vec{F}(x, y)$ is influenced by the details of the contacts and by the aspect ratio of the device. The 
component of $\vec{F}(x, y)$ along the drift velocity of each charge (and thus along $\vec{E}(x, y)$ ) represents the weight with which the carrier motion in each point of the device contributes to the total current, thus being analogous to the sensitivity coefficient introduced by Vandamme et al. [78-80]. Since for the purpose of the present study the relative weight of the contributions from the different regions of the device is not essential, we will assume a constant $\vec{F}(x, y)$ (equal to $1 / L$ and parallel to $\vec{E}(x, y)$ ), i.e., a uniform sensitivity coefficient.

In this approximation, the instantaneous current at the contacts is given by [72]

$$
i=\frac{1}{L} \int_{A} q\left(\mu_{n} n+\mu_{p} p\right) E d x d y .
$$

In the case of three-dimensional devices, this integral has to be replaced with an integral over the volume $V$, and $n$ and $p$ become volumetric densities (instead of surface densities). The average current can be written as

$$
I=q\left(\mu_{n} n+\mu_{p} p\right) E W .
$$

If, as it is usually the case [71], the effect of trapping and detrapping events on carrier densities is by far prevalent with respect to that on the mobilities and on the electric field, we can express the current fluctuations due to carriers being trapped and detrapped as

$$
\Delta i=\frac{1}{L} \int_{A} q\left(\mu_{n} \Delta n+\mu_{p} \Delta p\right) E d x d y
$$

where $\Delta n$ and $\Delta p$ are the fluctuations of electron and hole concentrations, respectively. Therefore, the ratio of the current fluctuation to the average current is equal to

$$
\begin{aligned}
\frac{\Delta i}{I} & =\frac{1}{A} \frac{\int_{A}\left(\mu_{n} \Delta n+\mu_{p} \Delta p\right) E d x d y}{\left(\mu_{n} n+\mu_{p} p\right) E} \\
& \simeq \frac{\mu_{n} \int_{A} \Delta n d x d y+\mu_{p} \int_{A} \Delta p d x d y}{A\left(\mu_{n} n+\mu_{p} p\right)} \\
& =\frac{\mu_{n} \Delta N+\mu_{p} \Delta P}{\mu_{n} N+\mu_{p} P},
\end{aligned}
$$

where the total number of electrons and holes in the area $A$ have been defined as $N=\int_{A} n d x d y$ and $P=\int_{A} p d x d y$, respectively, while their variations as an effect of the trapping-detrapping phenomena have been indicated with $\Delta N$ and $\Delta P$.

In order to compute $\Delta N$ and $\Delta P$, we use the following approach [72] (which differs from that used in Ref. [71] but leads to similar results). Let us consider a single trap, and let us define $\chi$ the number ( 0 or 1$)$ of electrons in the

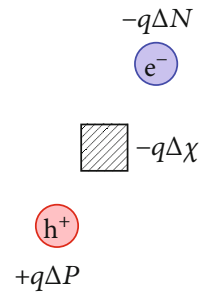

FIgURE 1: Variation $-q \Delta \chi$ of the charge in the considered trap (indicated with a square) and corresponding variations $-q \Delta N$ and $+q \Delta P$ of the charge due to the electrons and the holes in the surrounding region, which screen its electrostatic effect.

energy level of the impurity affected by the trapping events. In the following, we will indicate with $\Delta$ the variations with respect to the values with no trapped electron (i.e., when no electron is trapped all the variations $\Delta$ are zero). When an electron or a hole is trapped, the number of the corresponding charge carriers varies instantaneously by one, but, over time intervals exceeding the thermal generation-recombination time, the mass action law and electroneutrality must be satisfied. Therefore, for a generic variation $\Delta \chi$ of the number $\chi$ of electrons in the trap ( $\Delta \chi=\chi$, since $\Delta \chi=0$ if no electron is trapped, while $\Delta \chi=1$ if one electron is trapped), the variations $\Delta N$ and $\Delta P$ of carriers in the device have to satisfy

$$
\left\{\begin{array}{l}
N \Delta P+P \Delta N=0, \\
\Delta P-\Delta N-\Delta \chi=0
\end{array}\right.
$$

The first relation of Equation (7) can be obtained differentiating the relation $P N=c$ (i.e., the mass action law with both members multiplied by the square of the area $A$ ). (Equivalently, it can be obtained noting that the mass action law has to be valid both before and after the examined trapping event. Therefore, both $(\Delta P+P)(\Delta N+N)=c$ and $P N=c$ have to be satisfied; subtracting the two equations and neglecting the second-order term $\triangle P \Delta N$ with respect to the other ones, the first relation of Equation (7) is obtained.) The quantity $c$ is a constant which generally depends on the type of semiconductor and on the temperature, and thus, it does not change as a consequence of trapping events. Even in the case of graphene, in which $c$ depends also on the position of the Fermi level [81], we can assume its variation due to a trapping event to be negligible.

The second relation of Equation (7) enforces the electroneutrality of the device: assuming that the overall device, including the bias electrodes, was neutral before the examined event, the total change of charge deriving from the trapping event has to be zero: $q \Delta P-q(\Delta N+\Delta \chi)=0$ (see Figure 1), where we have included the variations in the number of free holes, of free electrons, and of electrons captured by the trap, which all contribute to the total charge. 
The system of Equation (7) has the following solutions:

$$
\begin{gathered}
\Delta N=-\frac{N}{P+N} \Delta \chi, \\
\Delta P=\frac{P}{P+N} \Delta \chi .
\end{gathered}
$$

In these solutions, the quantities $N$ and $P$ appear only through their ratio; therefore, the exact choice of the area where $N$ and $P$ are evaluated is irrelevant.

As can be seen from Equations (7) and (8), the necessity to satisfy both the mass action law and electroneutrality uniquely determines the values of the variations $\Delta N$ and $\Delta P$ deriving from a trapping event: these electron and hole fluctuations are therefore fully correlated.

Substituting these expressions into Equation (6), we obtain

$$
\begin{aligned}
\frac{\Delta i}{I} & =\frac{\mu_{n}(\Delta N / \Delta \chi)+\mu_{p}(\Delta P / \Delta \chi)}{A\left(\mu_{n} n+\mu_{p} p\right)} \Delta \chi \\
& =\frac{1}{A\left(\mu_{n} n+\mu_{p} p\right)} \frac{\mu_{p} P-\mu_{n} N}{P+N} \Delta \chi .
\end{aligned}
$$

Therefore, for a single trap, we have $\left(S_{I}\right.$ is the flicker noise power spectral density):

$$
\begin{aligned}
\frac{S_{I}}{I^{2}} & =\left(\frac{\mu_{n}(\Delta N / \Delta \chi)+\mu_{p}(\Delta P / \Delta \chi)}{A\left(\mu_{n} n+\mu_{p} p\right)}\right)^{2} S_{\chi} \\
& =\left(\frac{1}{A\left(\mu_{n} n+\mu_{p} p\right)} \frac{\mu_{p} P-\mu_{n} N}{P+N}\right)^{2} S_{\chi}
\end{aligned}
$$

where, neglecting the contribution of the average value, $S_{\chi}$ has a Lorentzian dependence on frequency, with a characteristic relaxation time (since $\Delta \chi$ is a random telegraph signal) [82].

If we suppose the many traps present in the device to be reciprocally independent, the overall noise spectrum can be obtained summing up their spectra. The combination of the effects of traps with properly distributed time constants leads then to $1 / f$ noise $[83,84]$ :

$$
\begin{aligned}
\frac{S_{I}}{I^{2}} & =\frac{\eta}{A}\left(\frac{\mu_{n}(\Delta N / \Delta \chi)+\mu_{p}(\Delta P / \Delta \chi)}{\mu_{n} n+\mu_{p} p}\right)^{2} \frac{1}{f^{\gamma}} \\
& =\frac{\eta}{A}\left(\frac{1}{\mu_{n} n+\mu_{p} p} \frac{\mu_{p} P-\mu_{n} N}{P+N}\right)^{2} \frac{1}{f^{\gamma}},
\end{aligned}
$$

with $\eta$ being a coefficient which depends on the concentration, distribution, and properties of the traps, while $\gamma$ is a number close to 1 .

We will report the flicker noise power spectral density as a function of $n-p$ (i.e., the charge density divided by $-q$ ), which in actual experiments is the quantity that can be adjusted by tuning the bias voltage of a gate capacitively coupled to the device.

From Equation (8), we can observe that if the electron concentration is much larger than that of holes, i.e., when $N \gg P$, we have $\Delta N \approx-\Delta \chi$ while $\Delta P \approx 0$, i.e., the variation in the trap charge is completely screened by electrons. In a similar way, when the hole concentration strongly dominates (i.e., $P \gg N$ ), $\Delta P \approx \Delta \chi$ and $\Delta N \approx 0$, which means that the trap charge variation is completely screened by holes. In intermediate conditions, the variation in the trap charge is screened by variations of both electron and hole concentrations. In particular, at the neutrality point (when $N=P$ ), the trap charge is screened for a half by a variation of the hole number and for the other half by an opposite variation of the electron number: $\Delta N=-\Delta \chi / 2$ and $\Delta P=\Delta \chi / 2$.

From Equation (11), we observe that the flicker noise power spectral density $S_{I}$ vanishes when $\mu_{p} P-\mu_{n} N=0$. The behavior of $S_{I}$ is symmetrical around this point if $\mu_{n}$ and $\mu_{p}$ coincide; otherwise, such a symmetry is absent. If $\mu_{n}=\mu_{p}$, the point in which $S_{I}$ vanishes and around which $S_{I}$ is symmetrical is the charge neutrality point (where $N-$ $P=0$ ); this condition is obtained for a Fermi energy corresponding to the middle of the gap between the conduction and valence bands if such bands are symmetric.

The quantities $N$ and $P$ depend, through the energy dispersion relations, on $E_{\mathrm{F}}$, i.e., on the relative position of the Fermi energy with respect to the local value of the potential energy. However, the nonuniform distribution of charged dopants and impurities (including the randomly located charged traps themselves) introduces a potential disorder (i.e., a random spatial variation of the potential energy) which can substantially alter this result. In order to introduce the effect of this random spatial energy variation in our calculations, for each value $E_{\mathrm{F}}$ of the Fermi energy we average our results over a Gaussian distribution of energies around $E_{\mathrm{F}}$ :

$$
\begin{aligned}
\frac{\left\langle S_{I}\right\rangle}{I^{2}} & =\frac{\eta}{A f^{\gamma}} \int_{-\infty}^{+\infty}\left(\frac{\mu_{n}\left((\Delta N / \Delta \chi)\left(E_{\mathrm{F}}+\varepsilon\right)\right)+\mu_{p}\left((\Delta P / \Delta \chi)\left(E_{\mathrm{F}}+\varepsilon\right)\right)}{\mu_{n} n\left(E_{\mathrm{F}}+\varepsilon\right)+\mu_{p} p\left(E_{\mathrm{F}}+\varepsilon\right)}\right)^{2} P(\varepsilon) d(\varepsilon) \\
& =\frac{\eta}{A f^{\gamma}} \int_{-\infty}^{+\infty}\left(\frac{1}{\mu_{n} n\left(E_{\mathrm{F}}+\varepsilon\right)+\mu_{p} p\left(E_{\mathrm{F}}+\varepsilon\right)} \frac{\mu_{p} P\left(E_{\mathrm{F}}+\varepsilon\right)-\mu_{n} N\left(E_{\mathrm{F}}+\varepsilon\right)}{P\left(E_{\mathrm{F}}+\varepsilon\right)+N\left(E_{\mathrm{F}}+\varepsilon\right)}\right)^{2} P(\varepsilon) d \varepsilon,
\end{aligned}
$$


where

$$
P(\varepsilon)=\frac{1}{\sqrt{2 \pi \sigma^{2}}} \exp \left(-\frac{\varepsilon^{2}}{2 \sigma^{2}}\right)
$$

represents a normalized Gaussian distribution with null average and standard deviation $\sigma$.

The standard deviation $\sigma$ of this Gaussian represents an estimation of the effect on the potential profile, and thus on the relative position $E_{\mathrm{F}}$ of the Fermi energy with respect to the potential energy, of the random charged impurities which represent the sources of the potential disorder. Therefore, the value of $\sigma$ does not depend only on the "strength" of the disorder sources but also on the electrostatic screening efficiency of the material, which can be estimated through the derivative $\partial(n-p) / \partial E_{\mathrm{F}}$ (or from the quantum capacitance, which is proportional to it [45]). If this derivative is larger, the same charged impurities induce a smaller variation of $E_{\mathrm{F}}$ (and thus a smaller $\sigma$ ), since this smaller variation of $E_{\mathrm{F}}$ is sufficient to screen the electrostatic effect of impurities through an opposite variation of the mobile charge density $-q(n-p)$.

When including potential disorder, we will report the flicker noise power spectral density as a function of $\langle n-p\rangle$, i.e., $n-p$ averaged over the same Gaussian distribution of energies:

$$
\langle n-p\rangle=\int_{-\infty}^{+\infty}\left(n\left(E_{\mathrm{F}}+\varepsilon\right)-p\left(E_{\mathrm{F}}+\varepsilon\right)\right) P(\varepsilon) d \varepsilon .
$$

The average over the potential disorder (see Equation (12)) decreases the dependence of the noise spectrum on the charge density, with a smoothing effect which increases with the disorder strength.

\section{Graphene}

First of all, let us analyze the case of monolayer and bilayer graphene (with Bernal stacking). In this material, the electron and hole bands are approximately symmetric and $\mu_{n}=\mu_{p}$. Therefore, Equation (6) becomes

$$
\frac{\Delta i}{I}=\frac{\Delta N+\Delta P}{N+P} .
$$

Analogous simplifications can be performed in Equations (9)-(12); for example, Equation (11) becomes

$$
\frac{S_{I}}{I^{2}}=\frac{\eta}{A}\left(\frac{1}{n+p} \frac{\Delta N+\Delta P}{\Delta \chi}\right)^{2} \frac{1}{f^{\gamma}}=\frac{\eta}{A}\left(\frac{1}{n+p} \frac{P-N}{P+N}\right)^{2} \frac{1}{f^{\gamma}} .
$$

For graphene, we compute the carrier concentrations $n$ and $p$ by integrating the product of the density of

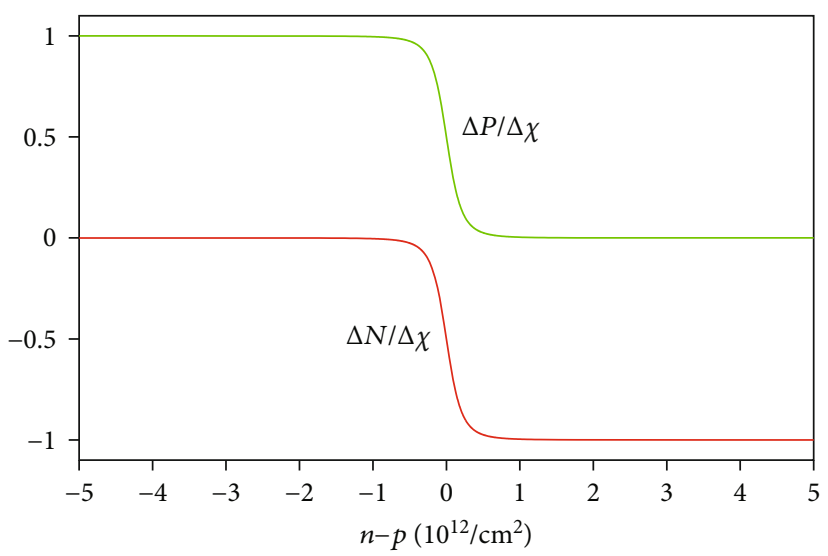

Figure 2: Behavior of $\Delta P / \Delta \chi$ and $\Delta N / \Delta \chi$ as a function of $n-p$, for monolayer graphene at $300 \mathrm{~K}$.

states and of the occupation function over the whole energy range:

$$
\begin{aligned}
& n=\int_{0}^{\infty} \operatorname{DOS}(E) f\left(E-E_{\mathrm{F}}\right) d E \\
& p=\int_{-\infty}^{0} \operatorname{DOS}(E)\left(1-f\left(E-E_{\mathrm{F}}\right)\right) d E,
\end{aligned}
$$

where DOS is the density of states and $f$ is the FermiDirac occupation function for electrons (therefore, $1-f$ is the occupation function for holes). The DOS, in turn, depends on the dispersion relations of monolayer graphene or bilayer graphene, i.e. $[14,18,53,54]$ :

$$
\begin{aligned}
& E_{m}(\kappa)= \pm \gamma \kappa, \\
& E_{b}(\kappa)= \pm\left(\frac{\gamma_{1}^{2}}{2}+\frac{\Delta^{2}}{4}+\gamma^{2} \kappa^{2}-\sqrt{\frac{\gamma_{1}^{4}}{4}+\left(\gamma_{1}^{2}+\Delta^{2}\right) \gamma^{2} \kappa^{2}}\right)^{1 / 2},
\end{aligned}
$$

where $\kappa$ is the difference between the wave vector and the Dirac point, $\gamma=\hbar v_{F}$ (with $\hbar$ the reduced Planck constant and $v_{\mathrm{F}}=8.73 \times 10^{5} \mathrm{~m} / \mathrm{s}$ the Fermi velocity of graphene), $\gamma_{1}=0.39 \mathrm{eV}$ is the graphene interlayer coupling, and $\Delta$ (the term which is responsible of the possible band gap opening) is the difference between the on-site energies in the two layers, which is approximately proportional to the carrier concentration $n-p$ (more in detail, $\Delta \approx 1.4 \times$ $\left.10^{-18} \mathrm{eV} \mathrm{m}^{2} \times(n-p)\right)$.

In Figures 2-5, we report some results obtained for graphene at $300 \mathrm{~K}$, without potential disorder averaging. Figures 2 and 3 refer to monolayer graphene, while Figures 4 and 5 refer to bilayer graphene.

In Figures 2 and 4, we report the quantities $\Delta P / \Delta \chi$ and $\Delta N / \Delta \chi$ (evaluated from Equation (8)) as a function of $n-p$ (for monolayer and bilayer graphene, respectively). As in the general case, the charge variation in the traps is mainly screened by holes when the holes are the dominant carriers, while it is mainly screened by electrons when the 


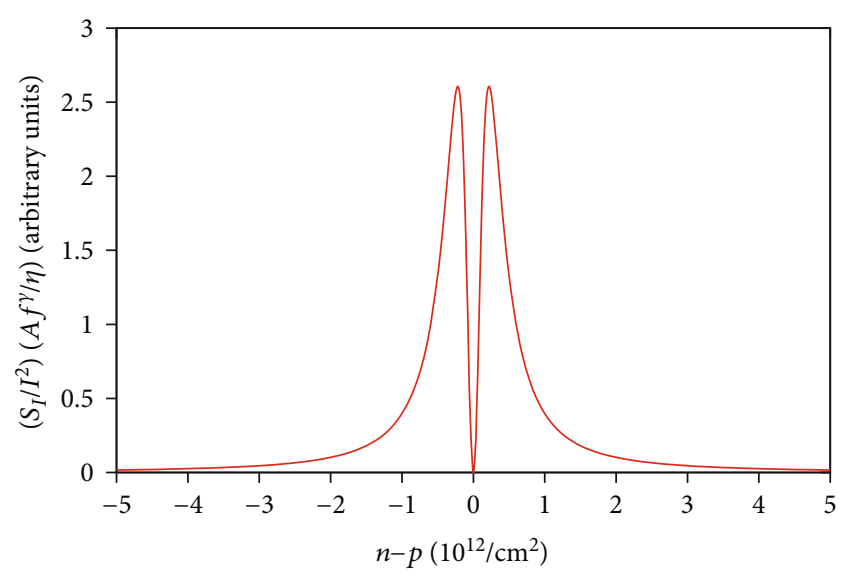

Figure 3: Behavior of $\left(S_{I} / I^{2}\right)\left(A f^{\gamma} / \eta\right)$ as a function of $n-p$, for monolayer graphene at $300 \mathrm{~K}$, in the absence of potential disorder.

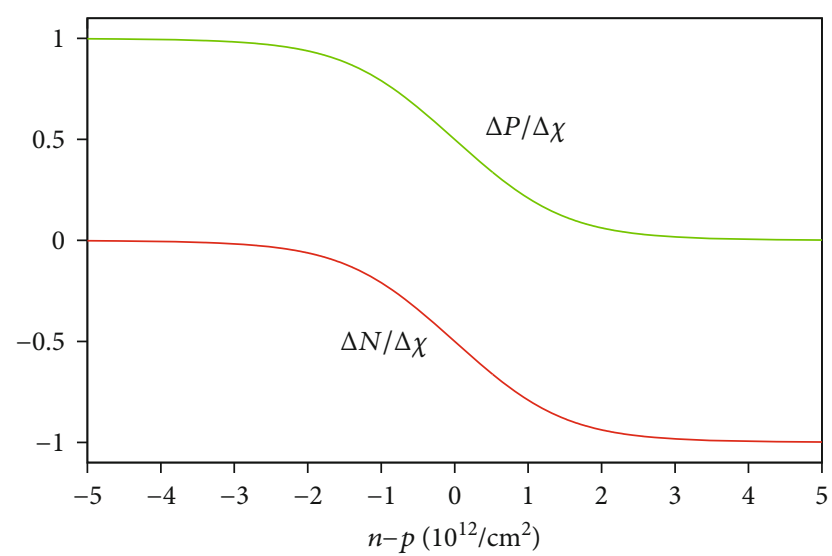

Figure 4: Behavior of $\Delta P / \Delta \chi$ and $\Delta N / \Delta \chi$ as a function of $n-p$, for bilayer graphene at $300 \mathrm{~K}$.

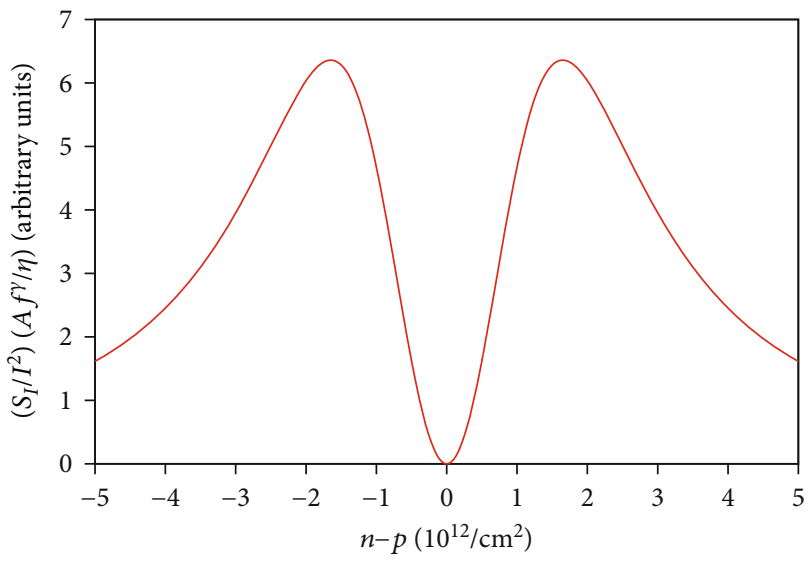

Figure 5: Behavior of $\left(S_{I} / I^{2}\right)\left(A f^{\gamma} / \eta\right)$ as a function of $n-p$, for bilayer graphene at $300 \mathrm{~K}$, in the absence of potential disorder.

electrons are the dominant carriers. It is instead symmetrically screened by electrons and holes in the charge neutrality point.

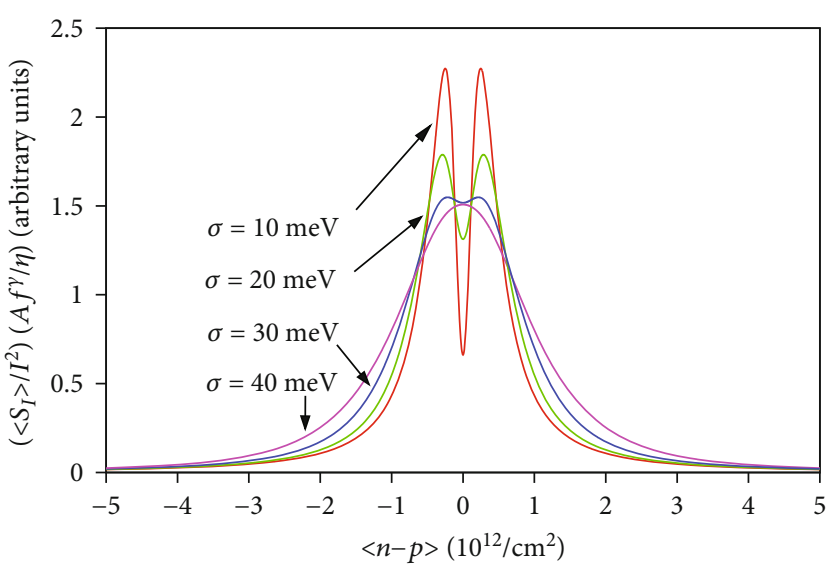

Figure 6: Behavior of $\left(\left\langle S_{I}\right\rangle / I^{2}\right)\left(A f^{\gamma} / \eta\right)$ as a function of $\langle n-p\rangle$, for monolayer graphene at $300 \mathrm{~K}$. The averages have been performed over four Gaussian distributions of potential disorder with standard deviations $\sigma=10,20,30$, and $40 \mathrm{meV}$, respectively.

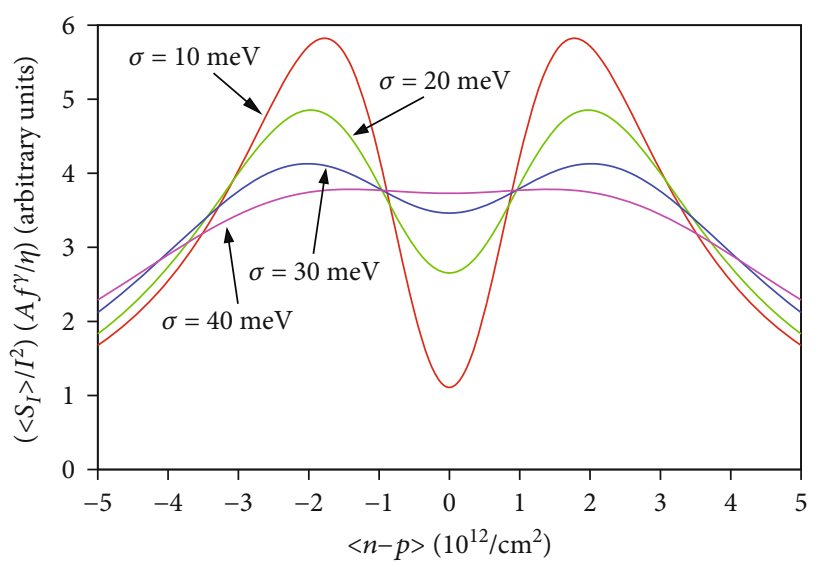

Figure 7: Behavior of $\left(\left\langle S_{I}\right\rangle / I^{2}\right)\left(A f^{\gamma} / \eta\right)$ as a function of $\langle n-p\rangle$, for bilayer graphene at $300 \mathrm{~K}$. The averages have been performed over four Gaussian distributions of potential disorder with standard deviations $\sigma=10,20,30$, and $40 \mathrm{meV}$, respectively.

In Figures 3 and 5, we report the quantity $\left(S_{I} / I^{2}\right)\left(A f^{\gamma} / \eta\right)$ (evaluated from Equation (11)) as a function of $n-p$ (for monolayer and bilayer graphene, respectively). Since in graphene $\mu_{n}=\mu_{p}$, the behavior of the flicker noise power spectral density is exactly symmetrical with respect to the neutrality point. In particular, in the charge neutrality point (Dirac point), where $\Delta N=-\Delta \chi / 2$ and $\Delta P=\Delta \chi / 2$, the fluctuation of the current (and therefore the noise power spectral density) completely vanishes (see Equation (16)). Comparing Figures 3 and 5, we notice that the dip around the Dirac point is wider in bilayer graphene than in monolayer graphene, as a consequence of their different dispersion relations.

In Figures 6 and 7, we show analogous results obtained averaging the spectrum of monolayer (Figure 6) and bilayer (Figure 7) graphene at $300 \mathrm{~K}$ over potential disorder. In particular, we report the value of $\left(\left\langle S_{I}\right\rangle / I^{2}\right)\left(A f^{\gamma} / \eta\right)$ (i.e., the integral appearing in Equation (12)) as a function of $\langle n-p\rangle$ (i.e., the charge density divided by the electron charge, 
averaged according to Equation (14)). Both for monolayer and bilayer graphene, we show the results for the following four different values of $\sigma: 10,20,30$, and $40 \mathrm{meV}$. In both cases, we observe that the minimum of the spectrum at the charge neutrality point tends to disappear increasing the strength of the disorder: therefore, the behavior of the flicker noise power spectral density evolves from an " $M$ "-shaped one to a " $\Lambda$ "-shaped one. However, as we can deduce from the corresponding values of $\partial(n-p) / \partial E_{\mathrm{F}}$ (which in the charge neutrality point is equal to about $7 \times 10^{12} \mathrm{~cm}^{-2} \mathrm{eV}^{-1}$ in monolayer graphene and to about $44 \times 10^{12} \mathrm{~cm}^{-2} \mathrm{eV}^{-1}$ in bilayer graphene), the screening of potential fluctuations is about six times larger in bilayer graphene than in monolayer graphene. This is a consequence of the different dispersion relations of the two materials, with bilayer graphene characterized by flatter bands near the Dirac points [50]. Therefore, for similar impurity distributions, values of $\sigma$ should be considered for monolayer graphene that are approximately six times larger than those considered for bilayer graphene. As a consequence, for realistic values of disorder, monolayer graphene does not usually exhibit a minimum of the flicker noise power spectral density at the charge neutrality point. On the contrary, in sufficiently clean samples of bilayer graphene, an "M"-shaped behavior is often observed, with a minimum at the Dirac point. A similar "M"-shaped behavior has been observed also in the case of suspended monolayer graphene, for which potential disorder is much weaker than for monolayer graphene on a substrate.

\section{Ordinary Semiconductors}

Let us now move on to the case of ordinary semiconductors, such as silicon and gallium arsenide, for which a minimum of the flicker noise power spectral density at the charge neutrality point has never been experimentally observed.

Also in this case, we can adopt the numerical procedure described in Section 2, substituting (in the case of 3dimensional channels) areas with volumes. In order to simplify the calculations and to reach general results, we neglect the details of the band structure of the materials and, assuming a constant effective mass, we use the semiclassical expressions for the carrier concentrations $[85,86]$ :

$$
\begin{aligned}
& n=N_{\mathrm{C}} \exp \left(-\frac{E_{\mathrm{C}}-E_{\mathrm{F}}}{k_{\mathrm{B}} T}\right), \\
& p=N_{\mathrm{V}} \exp \left(-\frac{E_{\mathrm{F}}-E_{\mathrm{V}}}{k_{\mathrm{B}} T}\right),
\end{aligned}
$$

where $E_{\mathrm{C}}$ is the conductance band minimum, $E_{\mathrm{V}}$ is the valence band maximum, $k_{\mathrm{B}}$ is the Boltzmann constant, and $T$ is the absolute temperature.

In particular, we perform our calculations using the material parameters for silicon and for gallium arsenide.

For silicon, we consider the following parameters: $N_{\mathrm{C}}=$ $2.82 \times 10^{19} \mathrm{~cm}^{-3}, N_{\mathrm{V}}=1.04 \times 10^{19} \mathrm{~cm}^{-3}, E_{\mathrm{G}}=1.12 \mathrm{eV}, \mu_{p}=$ $450 \mathrm{~cm}^{2} /(\mathrm{V} \mathrm{s})$, and $\mu_{n}=1400 \mathrm{~cm}^{2} /(\mathrm{V} \mathrm{s})$.

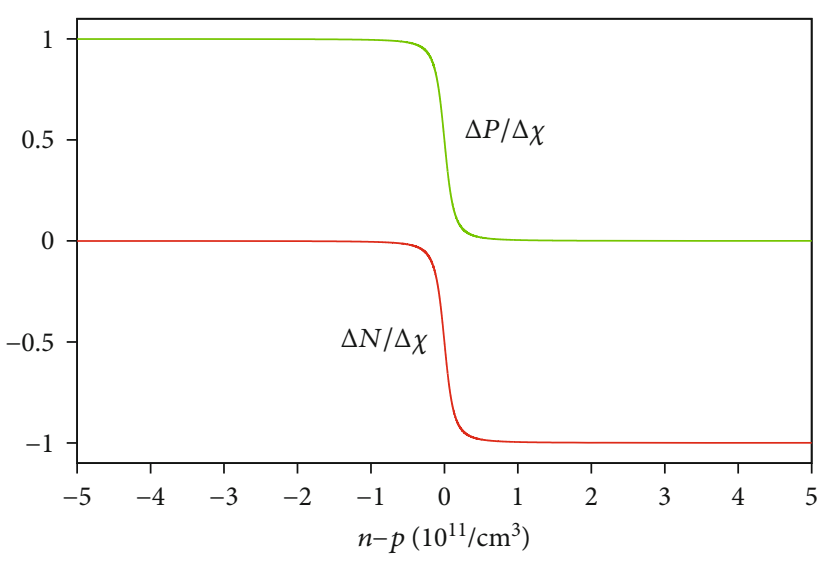

Figure 8: Behavior of $\Delta P / \Delta \chi$ and $\Delta N / \Delta \chi$ as a function of $n-p$, for silicon at $300 \mathrm{~K}$.

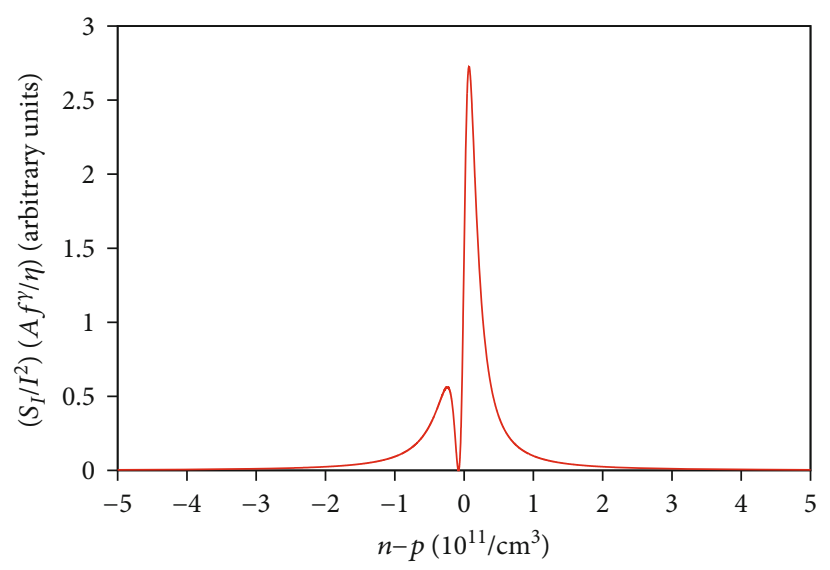

Figure 9: Behavior of $\left(S_{I} / I^{2}\right)\left(A f^{\gamma} / \eta\right)$ as a function of $n-p$, for silicon at $300 \mathrm{~K}$, in the absence of potential disorder.

Instead, for gallium arsenide, we assume the following: $N_{\mathrm{C}}=4.7 \times 10^{17} \mathrm{~cm}^{-3}, \quad N_{\mathrm{V}}=7.0 \times 10^{18} \mathrm{~cm}^{-3}, \quad E_{\mathrm{G}}=1.42 \mathrm{eV}$, $\mu_{p}=400 \mathrm{~cm}^{2} /(\mathrm{V} \mathrm{s})$, and $\mu_{n}=8500 \mathrm{~cm}^{2} /(\mathrm{V} \mathrm{s})$.

In Figures 8 and 9, we report the results obtained for silicon at $300 \mathrm{~K}$ without potential disorder averaging. More in detail, in Figure 8, we show the ratios $\Delta P / \Delta \chi$ and $\Delta N / \Delta \chi$ (evaluated according to Equation (8)) as a function of $n-\mathrm{p}$, while in Figure 9, we report the behavior of the quantity $\left(S_{I}\right.$ $\left./ I^{2}\right)\left(A f^{\gamma} / \eta\right)$ (evaluated according to Equation (11)) as a function of $n-p$.

In Figures 10 and 11, we report the analogous results for gallium arsenide at $300 \mathrm{~K}$ without potential disorder averaging.

As we see from the reported parameters, contrary to graphene, these semiconductors have quite different mobilities for electrons and holes. This is the reason of the clear asymmetry observed in the results, with a larger noise spectrum in the region where transport is dominated by electrons, i.e., the carriers with higher mobility. We can also observe that the asymmetry is stronger in gallium arsenide, for which the difference between electron and hole mobility is larger. Due to 


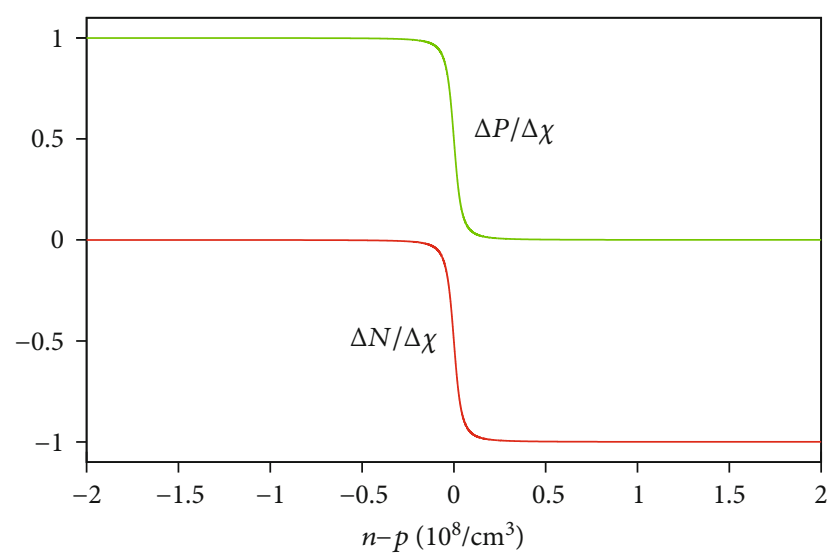

FIgURE 10: Behavior of $\Delta P / \Delta \chi$ and $\Delta N / \Delta \chi$ as a function of $n-p$, for gallium arsenide at $300 \mathrm{~K}$.

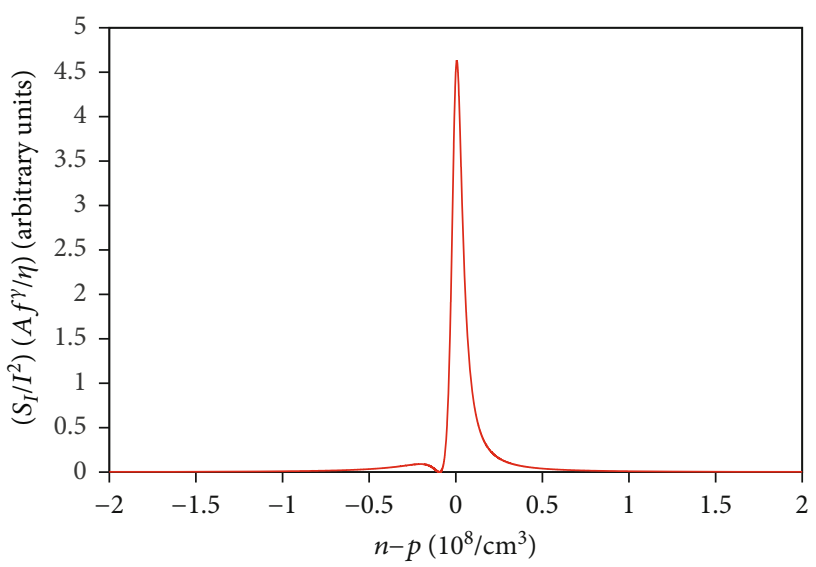

Figure 11: Behavior of $\left(S_{I} / I^{2}\right)\left(A f^{\gamma} / \eta\right)$ as a function of $n-p$, for gallium arsenide at $300 \mathrm{~K}$, in the absence of potential disorder.

the difference in mobility, the spectrum does not vanish any more at the charge neutrality point (where $n-p=0$ ) but where $\mu_{p} P-\mu_{n} N=0$. Moreover, with respect to graphene (for which, in the absence of disorder, this vanishing point presented a sharp reduction of the spectrum with respect to both the hole-dominated and the electron-dominated conduction regions), here, due to the spectrum asymmetry, the minimum point is much less prominent. Indeed, especially in the case of gallium arsenide, it is nearly indistinguishable from the hole-dominated region, where the spectrum is already very low.

Therefore, when the effect of potential disorder is introduced, by averaging over a Gaussian energy distribution with standard deviation $\sigma$ according to Equation (12), the local minimum of the spectrum completely disappears and the spectrum exhibits a " $\Lambda$ " shape, with a maximum centered in the electron-dominated region, where the mobility is larger. The behavior of $\left(\left\langle S_{I}\right\rangle / I^{2}\right)\left(A f^{\gamma} / \eta\right)$ (i.e., the integral appearing in Equation (12)) as a function of $\langle n-p\rangle$ (given by Equation (14)) is reported in Figures 12 and 13, at $300 \mathrm{~K}$, for silicon and for gallium arsenide, respectively, for four different values of $\sigma: 10,20,30$, and $40 \mathrm{meV}$. The described behavior is observed for both semiconductors but

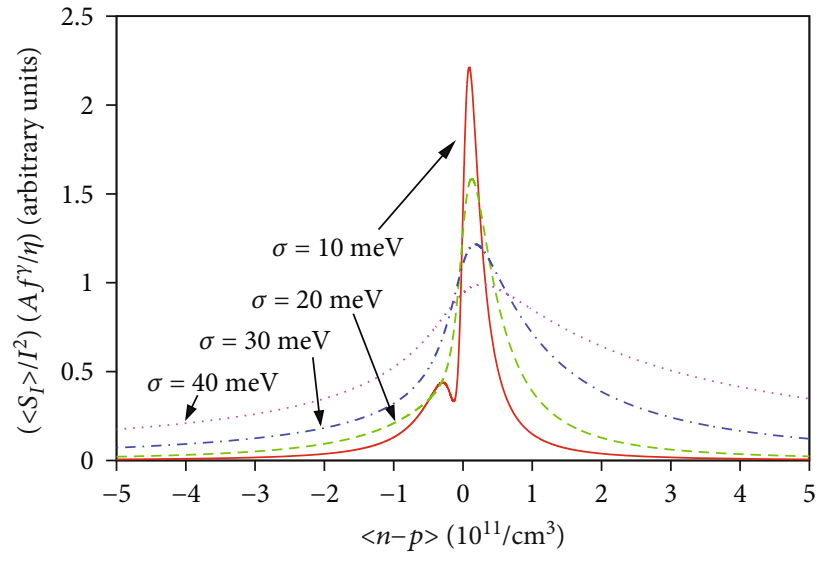

FIgURE 12: Behavior of $\left(\left\langle S_{I}\right\rangle / I^{2}\right)\left(A f^{\gamma} / \eta\right)$ as a function of $\langle n-p\rangle$, for silicon at $300 \mathrm{~K}$. The averages have been performed over four Gaussian distributions of potential disorder with standard deviations $\sigma=10,20,30$, and $40 \mathrm{meV}$, respectively.

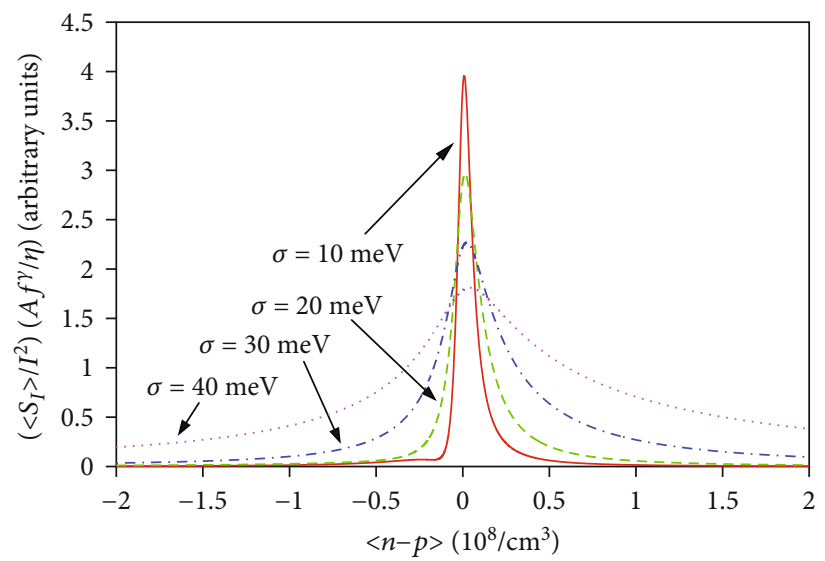

FIgURE 13: Behavior of $\left(\left\langle S_{I}\right\rangle / I^{2}\right)\left(A f^{\gamma} / \eta\right)$ as a function of $\langle n-p\rangle$, for gallium arsenide at $300 \mathrm{~K}$. The averages have been performed over four Gaussian distributions of potential disorder with standard deviations $\sigma=10,20,30$, and $40 \mathrm{meV}$, respectively.

is even more apparent in the case of gallium arsenide, for which a minimum in the spectrum was already hard to recognize in the absence of disorder.

The fact that in a real three-dimensional material it is difficult, if not impossible, to modulate the position of the Fermi level uniformly in the whole volume, further prevents a perfect balance of electron and hole current fluctuations in the overall device and thus makes it impossible to experimentally observe a flicker noise cancellation analogous to that described for graphene.

\section{Conclusions}

Exploiting a model based on charge neutrality and on the mass action law, we have compared the flicker noise behavior of graphene and of more common semiconductors, such as silicon and gallium arsenide. We have concluded that a minimum of the flicker noise power spectral density can be 
observed at the charge neutrality point only in materials where electrons and holes have a highly symmetric transport behavior, in particular have approximately the same mobility (the case of graphene), and only if the potential disorder is low. When these conditions are satisfied, the opposite variations of electron and hole concentrations induced by trapping phenomena reciprocally cancel, leading to no current fluctuations. Instead, in the presence of electron/hole asymmetry (the case of ordinary semiconductors) or of a significant degree of potential disorder, such a minimum disappears.

We believe that this analysis, clarifying the mechanisms that cause or prevent a reduction, at proper bias conditions, of the intrinsic device flicker noise, can be useful for the design of low-noise devices and in particular of highsensitivity sensors.

\section{Data Availability}

The numerical data used to support the findings of this study are available from the corresponding author upon request.

\section{Conflicts of Interest}

The authors declare no conflict of interest.

\section{Acknowledgments}

This work was partially supported by the Italian Ministry of Education and Research (MIUR) in the framework of the CrossLab project (Departments of Excellence).

\section{References}

[1] G. Schmera, C. Kwan, P. Ajayan, R. Vajtai, and L. B. Kish, "Fluctuation-enhanced sensing: status and perspectives," IEEE Sensors Journal, vol. 8, no. 6, pp. 714-719, 2008.

[2] A. Van der Ziel, Noise in Solid State Devices and Circuits, Wiley-Interscience, New York, NY, USA, 1986.

[3] A. Van der Ziel, Noise in Measurements, Wiley, New York, NY, USA, 1976.

[4] C. D. Motchenbacher and J. A. Connelly, Low-Noise Electronic System Design, Wiley-Interscience, New York, NY, USA, 1993.

[5] Y. M. Blanter and M. Büttiker, "Shot noise in mesoscopic conductors," Physics Reports, vol. 336, no. 1-2, pp. 1-166, 2000.

[6] F. N. Hooge, T. G. M. Kleinpenning, and L. K. J. Vandamme, "Experimental studies on 1/f noise," Reports on Progress in Physics, vol. 44, no. 5, pp. 479-532, 1981.

[7] M. Macucci and P. Marconcini, "Origin of shot noise in mesoscopic cavities," Fluctuation and Noise Letters, vol. 15, no. 3, p. 1640006, 2016.

[8] M. Macucci and P. Marconcini, "Shot noise suppression due to a magnetic field in disordered conductors," Journal of Computational Electronics, vol. 14, no. 1, pp. 107-113, 2015.

[9] P. Marconcini, M. Totaro, G. Basso, and M. Macucci, "Effect of potential fluctuations on shot noise suppression in mesoscopic cavities," AIP Advances, vol. 3, no. 6, article 062131, 2013.

[10] F. N. Hooge, " $1 / f$ noise is no surface effect," Physics Letters A, vol. 29, no. 3, pp. 139-140, 1969.
[11] A. Van der Ziel, "Flicker noise in electronic devices," in Advances in Electronics and Electron Physics, L. Marton and C. Marton, Eds., vol. 49, pp. 225-297, Academic Press, New York, NY, USA, 1979.

[12] F. Hofman and R. J. J. Zijlstra, “The validity of Hooge's law for 1/f noise," Solid State Communications, vol. 72, no. 12, pp. 1163-1166, 1989.

[13] K. S. Novoselov, A. K. Geim, S. V. Morozov et al., "Electric field effect in atomically thin carbon films," Science, vol. 306, no. 5696, pp. 666-669, 2004.

[14] A. H. Castro Neto, F. Guinea, N. M. R. Peres, K. S. Novoselov, and A. K. Geim, "The electronic properties of graphene," Reviews of Modern Physics, vol. 81, no. 1, pp. 109-162, 2009.

[15] M. I. Katsnelson, Graphene: Carbon in Two Dimensions, Cambridge University Press, Cambridge, UK, 2012.

[16] L. E. F. Foa Torres, S. Roche, and J.-C. Charlier, Introduction to Graphene-Based Nanomaterials: From Electronic Structure to Quantum Transport, Cambridge University Press, Cambridge, UK, 2014.

[17] T. Enoki and T. Ando, Physics and Chemistry of Graphene: Graphene to Nanographene, Pan Stanford, Boca Raton, FL, USA, 2014.

[18] P. Marconcini and M. Macucci, “The k.p method and its application to graphene, carbon nanotubes and graphene nanoribbons: the Dirac equation," La Rivista del Nuovo Cimento, vol. 34, no. 8-9, pp. 489-584, 2011.

[19] T. Ando, "Theory of electronic states and transport in carbon nanotubes," Journal of the Physical Society of Japan, vol. 74, no. 3, pp. 777-817, 2005.

[20] L. Brey and H. A. Fertig, "Electronic states of graphene nanoribbons studied with the Dirac equation," Physical Review B, vol. 73, no. 23, p. 235411, 2006.

[21] Y.-W. Son, M. L. Cohen, and S. G. Louie, "Energy gaps in graphene nanoribbons," Physical Review Letters, vol. 97, no. 21, p. 216803, 2006.

[22] D. Logoteta, P. Marconcini, C. Bonati, M. Fagotti, and M. Macucci, "High-performance solution of the transport problem in a graphene armchair structure with a generic potential," Physical Review E, vol. 89, no. 6, article 063309, 2014.

[23] G. Gui, J. Li, and J. Zhong, "Band structure engineering of graphene by strain: first-principles calculations," Physical Review $B$, vol. 78, no. 7, article 075435, 2008.

[24] Z. H. Ni, T. Yu, Y. H. Lu, Y. Y. Wang, Y. P. Feng, and Z. X. Shen, "Uniaxial strain on graphene: Raman spectroscopy study and band-gap opening," ACS Nano, vol. 2, no. 11, pp. 2301-2305, 2008.

[25] P. Marconcini, A. Cresti, F. Triozon et al., "Atomistic borondoped graphene field-effect transistors: a route toward unipolar characteristics," ACS Nano, vol. 6, no. 9, pp. 7942-7947, 2012.

[26] P. Marconcini, A. Cresti, and S. Roche, "Effect of the channel length on the transport characteristics of transistors based on boron-doped graphene ribbons," Materials, vol. 11, no. 5, p. 667, 2018.

[27] D. Wei, Y. Liu, Y. Wang, H. Zhang, L. Huang, and G. Yu, "Synthesis of $\mathrm{N}$-doped graphene by chemical vapor deposition and its electrical properties," Nano Letters, vol. 9, no. 5, pp. 1752$1758,2009$. 
[28] D. W. Boukhvalov and M. I. Katsnelson, "Tuning the gap in bilayer graphene using chemical functionalization: density functional calculations," Physical Review B, vol. 78, no. 8, article 085413, 2008.

[29] D. C. Elias, R. R. Nair, T. M. G. Mohiuddin et al., "Control of graphene's properties by reversible hydrogenation: evidence for graphane," Science, vol. 323, no. 5914, pp. 610-613, 2009.

[30] J. A. Fürst, J. G. Pedersen, C. Flindt et al., "Electronic properties of graphene antidot lattices," New Journal of Physics, vol. 11, no. 9, article 095020, 2009.

[31] P. Marconcini and M. Macucci, "Envelope-function based transport simulation of a graphene ribbon with an antidot lattice," IEEE Transactions on Nanotechnology, vol. 16, no. 4, pp. 534-544, 2017.

[32] K. S. Novoselov, A. Mishchenko, A. Carvalho, and A. H. Castro Neto, "2D materials and van der Waals heterostructures," Science, vol. 353, no. 6298, p. aac9439, 2016.

[33] M. Xu, T. Liang, M. Shi, and H. Chen, "Graphene-like twodimensional materials," Chemical Reviews, vol. 113, no. 5, pp. 3766-3798, 2013.

[34] L. Jiang, P. Marconcini, M. S. Hossian et al., "A tight binding and $\mathrm{k} \cdot \mathrm{p}$ study of monolayer stanene," Scientific Reports, vol. 7, no. 1, p. 12069, 2017.

[35] A. K. Geim and K. S. Novoselov, "The rise of graphene," Nature Materials, vol. 6, no. 3, pp. 183-191, 2007.

[36] K. S. Novoselov, V. I. Fal'ko, L. Colombo, P. R. Gellert, M. G. Schwab, and K. Kim, "A roadmap for graphene," Nature, vol. 490, no. 7419, pp. 192-200, 2012.

[37] Y. Zhu, H. Ji, H.-M. Cheng, and R. S. Ruoff, "Mass production and industrial applications of graphene materials," National Science Review, vol. 5, no. 1, pp. 90-101, 2018.

[38] S. Nazarpour and S. R. Waite, Graphene Technology: From Laboratory to Fabrication, Wiley-VCH, Weinheim, Germany, 2016.

[39] M. Sharon and M. Sharon, Graphene: An Introduction to the Fundamentals and Industrial Applications, John Wiley \& Sons and Scrivener Publishing LLC, Hoboken, New Jersey, USA: Salem, Massachusetts, USA, 2015.

[40] F. Schwierz, "Graphene transistors: status, prospects, and problems," Proceedings of the IEEE, vol. 101, no. 7, pp. 15671584, 2013.

[41] L. Liao and X. Duan, "Graphene for radio frequency electronics," Materials Today, vol. 15, no. 7-8, pp. 328-338, 2012.

[42] P. Marconcini and M. Macucci, "Symmetry-dependent transport behavior of graphene double dots," Journal of Applied Physics, vol. 114, no. 16, p. 163708, 2013.

[43] E. D. Herbschleb, R. K. Puddy, P. Marconcini et al., "Direct imaging of coherent quantum transport in graphene $\mathrm{p}-\mathrm{n}-\mathrm{p}$ junctions," Physical Review B, vol. 92, no. 12, p. 125414, 2015.

[44] P. Marconcini and M. Macucci, "Numerical analysis of the resistance behavior of an electrostatically-induced graphene double junction," Journal of Computational Electronics, vol. 14, no. 3, pp. 653-660, 2015.

[45] M. Macucci and P. Marconcini, "Approximate calculation of the potential profile in a graphene-based device," IET Circuits, Devices \& Systems, vol. 9, no. 1, pp. 30-38, 2015.

[46] P. Marconcini and M. Macucci, "Geometry-dependent conductance and noise behavior of a graphene ribbon with a series of randomly spaced potential barriers," Journal of Applied Physics, vol. 125, no. 24, p. 244302, 2019.
[47] M. Fagotti, C. Bonati, D. Logoteta, P. Marconcini, and M. Macucci, "Armchair graphene nanoribbons: PTsymmetry breaking and exceptional points without dissipation," Phys. Rev. B, vol. 83, no. 24, article 241406, 2011.

[48] M. I. Katsnelson and K. S. Novoselov, "Graphene: new bridge between condensed matter physics and quantum electrodynamics," Solid State Communications, vol. 143, no. 1-2, pp. 3-13, 2007.

[49] A. Shytov, M. Rudner, N. Gu, M. Katsnelson, and L. Levitov, "Atomic collapse, Lorentz boosts, Klein scattering, and other quantum-relativistic phenomena in graphene," Solid State Communications, vol. 149, no. 27-28, pp. 1087-1093, 2009.

[50] M. I. Katsnelson, K. S. Novoselov, and A. K. Geim, "Chiral tunnelling and the Klein paradox in graphene," Nature Physics, vol. 2, no. 9, pp. 620-625, 2006.

[51] K. S. Novoselov, A. K. Geim, S. V. Morozov et al., "Twodimensional gas of massless Dirac fermions in graphene," Nature, vol. 438, no. 7065, pp. 197-200, 2005.

[52] D. P. DiVincenzo and E. J. Mele, "Self-consistent effectivemass theory for intralayer screening in graphite intercalation compounds," Physical Review B, vol. 29, no. 4, pp. 16851694, 1984.

[53] E. McCann, "Asymmetry gap in the electronic band structure of bilayer graphene," Physical Review B, vol. 74, no. 16, article 161403, 2006.

[54] E. McCann and M. Koshino, "The electronic properties of bilayer graphene," Reports on Progress in Physics, vol. 76, no. 5, p. 056503, 2013.

[55] A. V. Rozhkov, A. O. Sboychakov, A. L. Rakhmanov, and F. Nori, "Electronic properties of graphene-based bilayer systems," Physics Reports, vol. 648, pp. 1-104, 2016.

[56] M. R. Connolly, R. K. Puddy, D. Logoteta et al., "Unraveling quantum hall breakdown in bilayer graphene with scanning gate microscopy," Nano Letters, vol. 12, no. 11, pp. 5448$5454,2012$.

[57] A. A. Balandin, "Low-frequency $1 / f$ noise in graphene devices," Nature Nanotechnology, vol. 8, no. 8, pp. 549-555, 2013.

[58] Y. Lin and P. Avouris, "Strong suppression of electrical noise in bilayer graphene nanodevices," Nano Letters, vol. 8, no. 8, pp. 2119-2125, 2008.

[59] Q. Shao, G. Liu, D. Teweldebrhan et al., "Flicker noise in bilayer graphene transistors," IEEE Electron Device Letters, vol. 30, no. 3, pp. 288-290, 2009.

[60] A. N. Pal, A. A. Bol, and A. Ghosh, "Large low-frequency resistance noise in chemical vapor deposited graphene," Applied Physics Letters, vol. 97, no. 13, p. 133504, 2010.

[61] S. Rumyantsev, G. Liu, W. Stillman, M. Shur, and A. A. Balandin, "Electrical and noise characteristics of graphene fieldeffect transistors: ambient effects, noise sources and physical mechanisms," Journal of Physics: Condensed Matter, vol. 22, no. 39, p. 395302, 2010.

[62] A. N. Pal, S. Ghatak, V. Kochat et al., "Microscopic mechanism of $1 / \mathrm{f}$ noise in graphene: role of energy band dispersion," ACS Nano, vol. 5, no. 3, pp. 2075-2081, 2011.

[63] A. N. Pal and A. Ghosh, "Ultralow noise field-effect transistor from multilayer graphene," Applied Physics Letters, vol. 95, no. 8 , article $082105,2009$.

[64] G. Xu, C. M. Torres Jr., Y. Zhang et al., "Effect of spatial charge inhomogeneity on $1 / f$ noise behavior in graphene," Nano Letters, vol. 10, no. 9, pp. 3312-3317, 2010. 
[65] I. Heller, S. Chatoor, J. Männik et al., "Charge noise in graphene transistors," Nano Letters, vol. 10, no. 5, pp. 15631567, 2010.

[66] Y. Zhang, E. E. Mendez, and X. Du, "Mobility-dependent lowfrequency noise in graphene field-effect transistors," ACS Nano, vol. 5, no. 10, pp. 8124-8130, 2011.

[67] A. N. Pal and A. Ghosh, "Resistance noise in electrically biased bilayer graphene," Physical Review Letters, vol. 102, no. 12, p. 126805, 2009.

[68] A. A. Kaverzin, A. S. Mayorov, A. Shytov, and D. W. Horsell, "Impurities as a source of $1 / f$ noise in graphene," Physical Review B, vol. 85, no. 7, article 075435, 2012.

[69] M. Z. Hossain, S. Rumyantsev, M. S. Shur, and A. A. Balandin, "Reduction of $1 / \mathrm{f}$ noise in graphene after electron-beam irradiation," Applied Physics Letters, vol. 102, no. 15, p. 153512, 2013.

[70] G. Liu, S. Rumyantsev, M. S. Shur, and A. A. Balandin, "Origin of $1 / \mathrm{f}$ noise in graphene multilayers: surface vs. volume," Applied Physics Letters, vol. 102, no. 9, p. 093111, 2013.

[71] B. Pellegrini, "1/f noise in graphene," The European Physical Journal B, vol. 86, no. 9, p. 373, 2013.

[72] B. Pellegrini, P. Marconcini, M. Macucci, G. Fiori, and G. Basso, "Carrier density dependence of $1 / f$ noise in graphene explained as a result of the interplay between band-structure and inhomogeneities," Journal of Statistical Mechanics: Theory and Experiment, vol. 2016, no. 5, article 054017, 2016.

[73] S. V. Vonsovsky and M. I. Katsnelson, Quantum Solid-State Physics, Springer, Berlin, Heidelberg, Germany, 1989.

[74] W. Shockley, "Currents to conductors induced by a moving point charge," Journal of Applied Physics, vol. 9, no. 10, pp. 635-636, 1938.

[75] S. Ramo, "Currents induced by electron motion," Proceedings of the IRE, vol. 27, no. 9, pp. 584-585, 1939.

[76] B. Pellegrini, "Electric charge motion, induced current, energy balance, and noise," Physical Review B, vol. 34, no. 8, pp. 59215924, 1986.

[77] B. Pellegrini, "Extension of the electrokinematics theorem to the electromagnetic field and quantum mechanics," Il Nuovo Cimento D, vol. 15, no. 6, pp. 855-879, 1993.

[78] L. K. J. Vandamme and W. M. G. van Bokhoven, "Conductance noise investigations with four arbitrarily shaped and placed electrodes," Applied Physics, vol. 14, no. 2, pp. 205215, 1977.

[79] L. K. J. Vandamme and A. H. de Kuijper, "Conductance noise investigations on symmetrical planar resistors with finite contacts," Solid State Electronics, vol. 22, no. 11, pp. 981-986, 1979.

[80] G. Leroy, J. Gest, L. K. J. Vandamme, and A. P. J. van Deursen, "Analytical expressions for the conductance noise measured with four circular contacts placed in a square array," Journal of Applied Physics, vol. 101, no. 6, article 063710, 2007.

[81] T. Fang, A. Konar, H. Xing, and D. Jena, "Carrier statistics and quantum capacitance of graphene sheets and ribbons," Applied Physics Letters, vol. 91, no. 9, p. 092109, 2007.

[82] S. Machlup, "Noise in semiconductors: spectrum of a twoparameter random signal," Journal of Applied Physics, vol. 25, no. 3, pp. 341-343, 1954.

[83] A. L. McWhorter, " $1 / f$ noise and germanium surface properties," in In Semiconductor Surface Physics, R. H. Kingston, Ed., pp. 207-228, University of Pennsylvania Press, Philadelphia, PA, USA, 1957.
[84] B. Pellegrini, “A general model of $1 / f^{\gamma}$ noise," Microelectronics Reliability, vol. 40, no. 11, pp. 1775-1780, 2000.

[85] S. M. Sze and K. K. Ng, Physics of Semiconductor Devices, Wiley, Hoboken, NJ, USA, 2007.

[86] S. O. Kasap, Principles of Electronic Materials and Devices, McGraw-Hill, New York, NY, USA, 2018. 